\title{
Sentinel lymph node biopsy in medullary thyroid carcinoma - a pilot study -
}

Authors:

Radan Dzodicl,2, Nada Santracl, Merima Orucil, Ivan Markovicl,2, Igor Djurisicle, Marko Buta', Gordana Pupic ${ }^{3}$

1 Institute for Oncology and Radiology of Serbia, Surgical Oncology Clinic, Pasterova 14, Belgrade, Serbia 2 University of Belgrade, School of Medicine, Dr Subotica 8, Belgrade, Serbia

${ }^{3}$ Institute for Oncology and Radiology of Serbia, Department of Pathology, Pasterova 14, Belgrade, Serbia

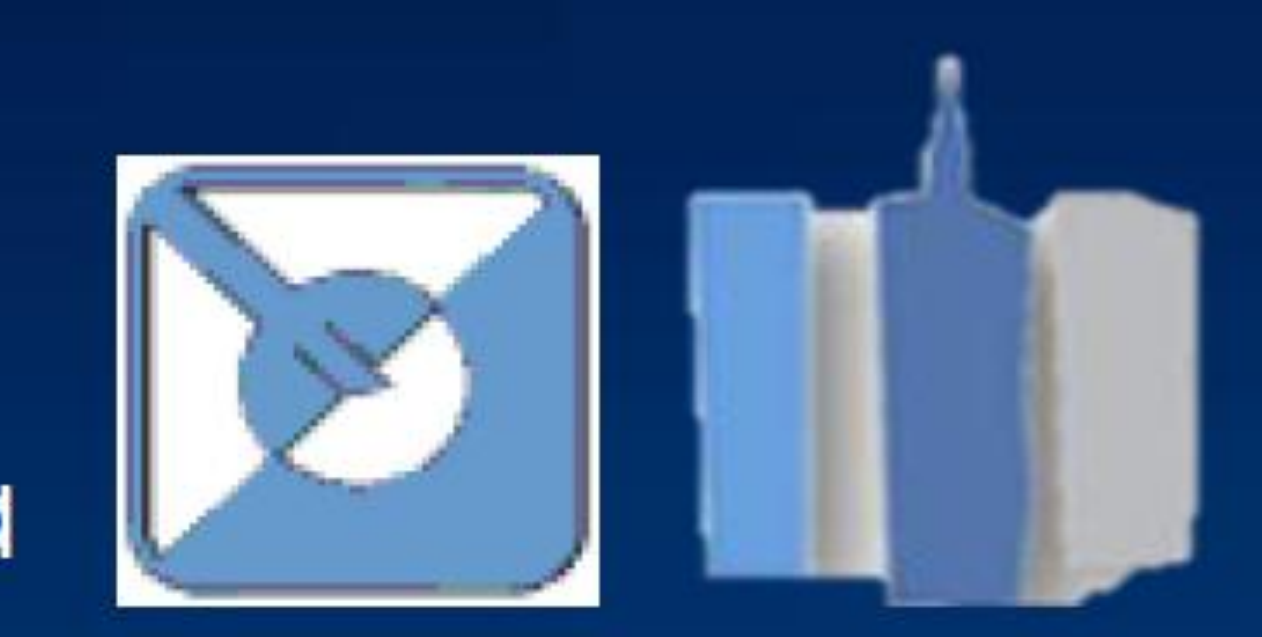

\section{INTRODUCTION}

Serum calcitonin levels (sCT) in medullary thyroid carcinoma (MTC) correlate with the tumor size and disease progression [1]. Lymph node (LN) metastases can be verified even in sCT lower than $100 \mathrm{pg} / \mathrm{ml}[2]$. The extent of LN dissection has not yet been standardized $[3,4]$.

The aim of this pilot study was to show our experience with sentinel lymph node (SLN) biopsy of jugulocarotid chain (JCC) in selection of patients with MTC for modified radical neck dissection (MRND).

\section{MATERIALS AND METHODS}

Following protocols of our institution, all patients with confirmed thyroid carcinoma on intraoperative frozen section analysis undergo total thyroidectomy, central neck dissection and SLN biopsy of JCC. SLN biopsy is performed after peritumoral injection of $1 \%$-methylene blue dye $(0.2-0.5 \mathrm{ml})$, and frozen section examination follows, in order to make decision on further MRND. For this study, we have selected 13 patients treated surgically from year 2007 to 2015 due to suspect MTC with increase of SCT, clinically and ultrasonically verified thyroid tumor and "clear" regional lymph nodes. Inclusion criteria for this pilot study were SCT lower than $1000 \mathrm{pg} / \mathrm{ml}$ and subcentimeter tumor size. All patients had uni- or bilateral SLN biopsy of supraomohyoid region (II-III). Besides these blue stained SLNs, we have removed surrounding LNs of II and III region in order to obtain more precise definite pathological evaluation of LNs.

\section{RESULTS}

In dissected central LNs, metastases were not verified. All SLNs were identified as benign, both on frozen section and definite pathological analysis, thus there were no false negative results. Besides SLNs, all LNs extirpated from II and III region were benign on definite pathology reports.

On postoperative check-ups, all sCT were in the normal range, patients suffered no complications of thyroid surgery and none have disease relapses. The SLN biopsy method's accuracy is $100 \%$.

\section{CONCLUSION}

This pilot study is the first reported experience with SLN biopsy of JCC in MTC using methylene blue dye. SLN biopsy can be precisely used for intraoperative assessment of lateral neck compartment. It enables adequate surgery for patients with subcentimeter MTC and SCT under 1000pg/ml, avoiding unnecessary prophylactic MRND.

\section{Figure: Blue-stained} sentinel lymph node identified in the right jugulocarotid region

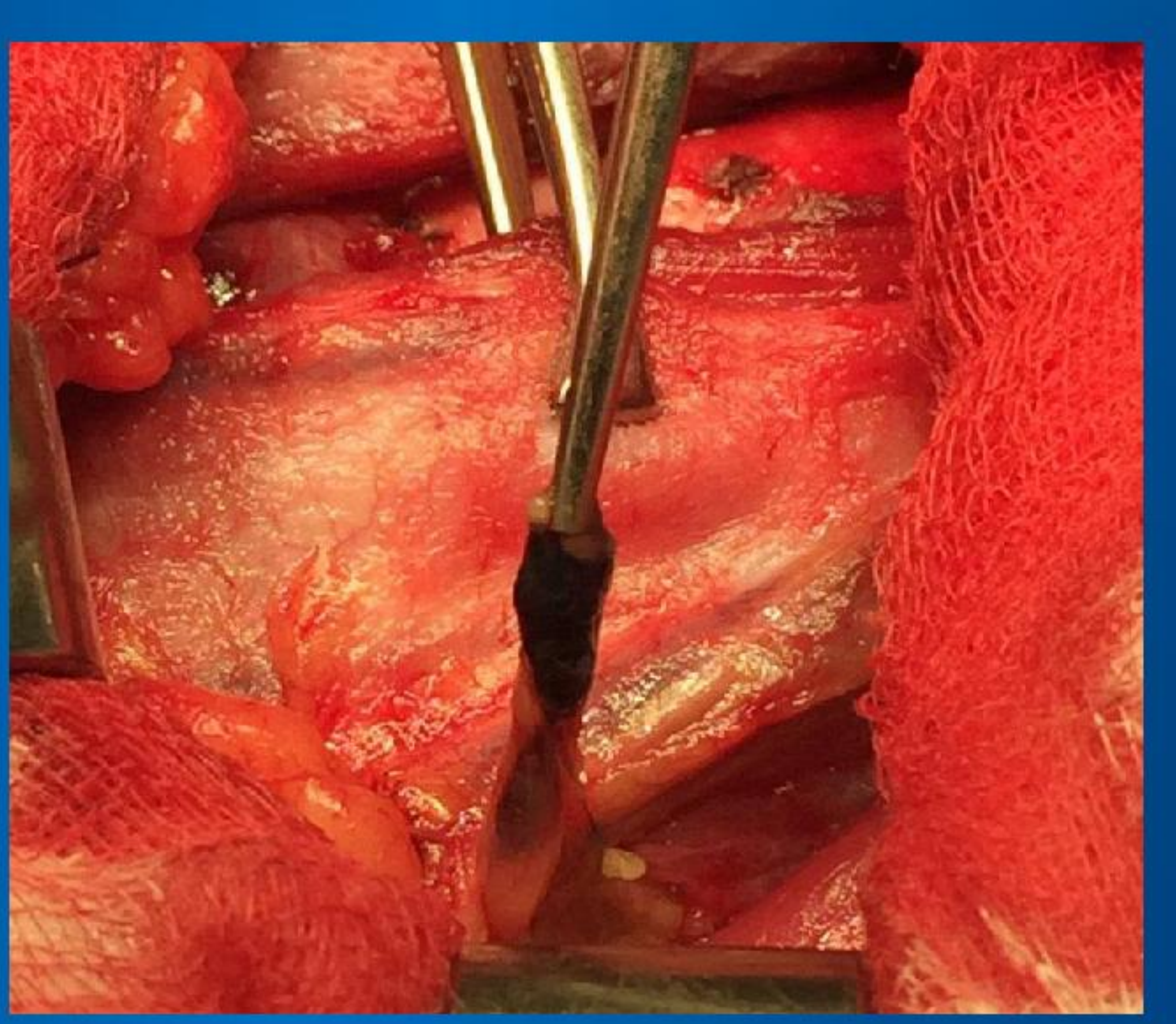

\section{REFERENCES}

Miyauchi A, Onishi T, Morimoto S, Takai S, Matsuzuka F, Kuma K, Maeda M, Kumahara Y. Relation of doubling time of plasma calcitonin levels to prognosis and recurrence of medullary thyroid carcinoma. Ann Surg 1984; 199(4): 461-466 Sippel RS, Kunnimalaiyaan M, Chen H. Current management of medullary thyroid cancer. Oncologist 2008;
13(5):539-547.

Dralle H, Damm I, Scheumann GF, Kotzerke J, Kupsch E, Geerlings H, Pichlmayr R. Compartment-oriented microdissection of regional lymph nodes in medullary thyroid carcinoma. Surg Today 1994; 24(2): 112-121.

Dzodic R, Markovic I, Inic M, Jokic N, Djurisic I, Zegarac M, Pupic G, Milovanovic Z, Jovic V, Jovanovic N. Sentinel lymph node biopsy may be used to support the decision to perform modified radical neck dissection in lymph node biopsy may be used to support the decision to pert
differentiated thyroid carcinoma. Wold J Surg 2006; 30(5): 841-846. 